\title{
Dynamics of magnetic modulation of ferrofluid droplets for digital microfluidic applications
}

\author{
Uddalok Sen $^{a}$, Souvick Chatterjee ${ }^{a}$, Swarnendu Sen ${ }^{b}$, Manish K. Tiwari ${ }^{c}$, Achintya \\ Mukhopadhyay $^{b}$, and Ranjan Ganguly ${ }^{d, 1}$ \\ ${ }^{a}$ Department of Mechanical and Industrial Engineering, University of Illinois at Chicago, Chicago, IL 60607, \\ USA \\ ${ }^{b}$ Mechanical Engineering Department, Jadavpur University, Kolkata 700032, India \\ ${ }^{c}$ Department of Mechanical Engineering, University College London, London WC1E 7JE, UK \\ ${ }^{d}$ Department of Power Engineering, Jadavpur University, Kolkata 700098, India
}

\begin{abstract}
Active control of droplet generation in a microfluidic platform attracts interest for development of digital microfluidic devices ranging from biosensors to micro-reactors to point-of-care diagnostic devices. The present paper characterises, through an unsteady three-dimensional Volume of Fluid (VOF) simulation, the active control of ferrofluid droplet generation in a microfluidic T-junction in presence of a non-uniform magnetic field created by an external magnetic dipole. Two distinctly different positions of the dipole were considered - one upstream of the junction and one downstream of the junction. While keeping the ferrofluid flow rate fixed, a parametric variation of the continuous phase capillary number, dipole strength, and dipole position was carried out. Differences in the flow behaviour in terms of dripping or jetting and the droplet characteristics in terms of droplet formation time period and droplet size were studied. The existence of a threshold dipole strength was identified, below which the magnetic force was not able to influence the flow behaviour. It was also observed that, for dipoles placed upstream, droplet formation was suppressed at some higher dipole strengths, and this value was found to increase with increasing capillary number. Droplet time period was also found to increase with increasing dipole strength, along with droplet size, i.e. an increase in droplet volume.
\end{abstract}

Keywords: Digital Microfluidics; Ferrofluid; Magnetic Actuation; Microfluidic T-junction; Droplet Generation

\section{Nomenclature}

\begin{tabular}{|llrl|}
\hline$b$ & Characteristic droplet width & $C a$ & Capillary number \\
$f$ & Volume fraction & $\mathbf{F}_{\mathbf{m}}$ & Magnetic Kelvin body force \\
$\mathbf{F}_{\mathbf{s}}$ & $h$ & Characteristic droplet height \\
$\mathbf{H}$ & Body force due to surface deformation & $m$ & Magnetic dipole strength \\
$\mathbf{M}$ & Magnetic field & $\hat{n}$ & Unit normal vector \\
$p$ & Magnetization & $\mathbf{r}$ & Position vector \\
$t$ & Pressure & $U_{c p}$ & Continuous phase velocity \\
$\mathbf{V}$ & Time & $V$ & Volume of droplet \\
$V_{0}$ & Velocity vector & $V_{e}$ & Percentage of excess volume of droplet \\
$x, y, z$ & Volume of droplet in non magnetic case & & \\
Greek Symbols & Coordinates & & \\
$\kappa$ & Curvature of the interface & $\mu$ & Viscosity \\
$\mu_{0}$ & Permeability of free space & $\rho$ & Density \\
$\sigma$ & Interfacial tension & $\tau$ & Droplet shedding time period \\
$\chi$ & Magnetic susceptibility & & \\
Subscripts & & & \\
$c$ & Continuous phase pressure probe & $d$ & Discrete phase pressure probe \\
1 & Continuous phase property & 2 & Discrete phase property \\
\hline
\end{tabular}

\section{Introduction}

The subdomain of microfluidics where fluid volumes are handled by manipulating discrete, individual droplets by means of an external field or actuation is termed 'digital microfluidics'. The advantage of digital microfluidics, in contrast to traditional continuous microfluidics, lies in the considerable reduction in the volume of analyte used. Moreover, difficulties in handling toxic material and cross-contamination in microchannels are also mitigated.

\footnotetext{
${ }^{1}$ Corresponding author, email:ranjan@pe.jusl.ac.in
} 


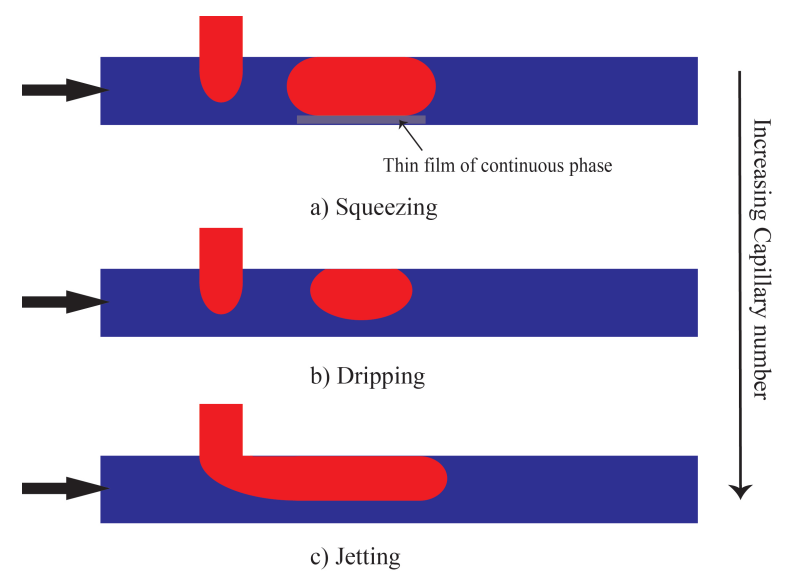

Figure 1: Schematic of the three distinct regimes of flow through a microfluidic T-junction

In digital microfluidic platforms, each droplet acts as an individual, isolated reaction chamber, thus resulting in the increased flexibility and programmability as compared to channel-based microfluidics. Thus, digital microfluidics can be used in applications where a high degree of flexibility is required [1]. These advantages have led to the successful integration of digital microfluidics in 'Lab-on-a-chip' applications. Significant application areas of digital microfluidics include proteomics [2], point-of-care diagnostics [3], molecular probe synthesis [4], immunoassays [5], cell culture [6], and chip-based PCR [7], among others [8-10].

For a functional digital microfluidic device, the two most important components are the droplet generation system and the droplet actuation system. From an operational point of view, microfluidic droplet generation configurations that are popular in the community include co-flow devices [11-13], flow-focusing devices [14-17], and T-junction [18-21]. The methods of manipulation of droplets in digital microfluidic devices can be diverse, such as electrical [22, 23], optic [24], electrophoretic [25] and dielectrophoretic [26], and magnetic [27, 28]. Ferrofluids provide a viable option for the choice of fluid in magnetically actuated digital microfluidic systems.

Ferrofluids are colloidal suspensions of single domain magnetic nanoparticles, typically of 5-15 nm diameter, containing $\mathrm{Ni}$, Co, $\mathrm{Mg}$, or $\mathrm{Zn}$ compositions of ferrite $\left(\mathrm{Fe}_{2} \mathrm{O}_{4}\right)$, magnetite $\left(\mathrm{Fe}_{3} \mathrm{O}_{4}\right)$, or maghemite $\left(\mathrm{g}-\mathrm{Fe}_{2} \mathrm{O}_{3}\right)$ in a nonmagnetic liquid (aqueous or hydrocarbon) carrier phase [29,30]. The particles at this size range exhibit superparamagnetic nature, implying that the particle magnetization curves do not show any hysteresis, although their magnetization is comparable to ferri- or ferromagnetic particles. While such a ferrofluid is nonmagnetic in the absence of a magnetic field, the magnetic moments of the superparamagnetic nanoparticles of ferrofluids are readily aligned (against the thermal Brownian disturbance) with an externally imposed magnetic field, making the fluid magnetically responsive. This feature is advantageous, since transport of individual ferrofluid droplets can be influenced by "action at a distance" in microfluidic environment either by an active device, e.g., a miniaturized permanent magnet or electromagnet, or by a passive device, e.g., a macro-scale biasing magnet in conjunction with micro-scale magnetizable elements for creating local field gradient. Ferrofluids have been extensively used in microfluidics [31], having diverse application areas.

In the context of droplet generation at a T-junction, the capillary number $\left(C a=\frac{\mu U_{c p}}{\sigma}\right.$, where $\mu$ and $U_{c p}$ are the viscosity and velocity of the continuous phase, and $\sigma$ is the interfacial tension) plays a key role. Existing literature $[18,32]$ shows that there are three major regimes of flow at a microfluidic T-junction: squeezing, dripping, and jetting (see the schematic diagram in Fig. 1). In the squeezing regime $\left(C a \leq 10^{-2}\right)$, interfacial force is much stronger and dominates over the shear force (Fig. 1(a)). The dispersed phase, in this regime, blocks off almost the entire cross-sectional area of the main channel, reducing the continuous phase to thin films between the dispersed phase and the walls of the channel. The resulting pressure difference squeezes the neck of the dispersed phase, thus forming droplets. Droplet volumes in the squeezing regime are governed by the ratio of the flow rates of the two fluids. At a higher value of $C a(\approx 0.025)$, the shear forces become significant for droplet generation, and this regime is known as the dripping regime (Fig. 1(b)). At an even higher $C a$, droplet formation is not observed. Instead, the dispersed phase enters the main channel and flows parallel to the continuous phase; the regime being known as jetting regime (Fig. 1(c)).

Ferrofluid droplet-based digital microfluidic platform has the unique advantage of magnetic manipulation of the dispersed magnetic phase to alter the droplet dynamics. However, dynamic interaction of magnetic, surface tension, and viscous forces makes investigation of such a system extremely complicated. Early work [33] on the dynamics of ferrofluid droplet breakup, as it passed through a narrow orifice, showed strong influence of orifice diameter on the droplet size and stretching length, while the number of total breaking droplets depended on the orifice diameter and local magnetic field. Sivasamy et al. [34] demonstrated CFD modeling of the droplet generation at a microfluidic T-junction using the VOF method; however, the effect of magnetic field was not 
Table 1: Properties of EFH3

\begin{tabular}{ll}
\hline Viscosity & $12 \times 10^{-3} \mathrm{~Pa} . \mathrm{s}$ \\
Density & $1.42 \times 10^{3} \mathrm{~kg} \cdot \mathrm{m}^{-3}$ \\
Saturation magnetization & $65 \mathrm{mT}$ \\
\hline
\end{tabular}

considered in that work. The literature, therefore, lacks in a comprehensive understanding of the different regimes of droplet generation and its interdependence with the applied magnetic field.

Digital microfluidics obviously has advantages of flexibility and precise control, but the robustness of channelbased microfluidics is unmatched. In the present work, an attempt has been made to bridge the two apparently contrasting aspects of robust droplet generation and precise control of dynamics inside a T-junction microchannel. Although ferrofluid droplet generation has been studied both experimentally [35-38] and numerically [39] in other droplet generating geometries such as flow-focusing, such an investigation was lacking in a T-junction geometry. A numerical investigation of ferrofluid droplet generation in a surfacted medium in a T-junction microchannel in the presence of an externally imposed magnetic field is carried out. The motivation of the present work lies in understanding the fundamental flow physics of droplet generation in a microfluidic T-junction. Even though such a configuration has been previously studied [40] experimentally, and it was reported that the magnetic force played a key role in the evolution of a droplet, important aspects like dripping-jetting transition could not be analysed. This transition is of particular importance in a digital microfluidic platform because it sets the limit for the maximum droplet volume that can be generated, which in turn governs the operating range of the devices. In this context, Tan et al. [40] have indicated there that a complex three dimensional numerical simulation with coupled fields is needed to gain insight into the droplet formation phenomenon in the presence of a magnetic field. Sivasamy et al. [34] presents a numerical investigation of the droplet formation phenomenon in a T-junction, but only in the absence of magnetic field. Therefore, a three dimensional numerical investigation of ferrofluid droplet formation in a T-junction in the presence of a non-uniform magnetic field has been attempted in the present work. Because of paucity of reliable literature data on ferrofluid properties, interfacial tension, and magnetic susceptibility are obtained from in-house experiments.

\section{Problem Description}

The computational geometry (Fig. 2) used in the present study is similar to the one used by Sivasamy et al. [34] in their experiments and simulations. A microfluidic channel of rectangular cross-section having length, width and height of $2000 \mu \mathrm{m}, 200 \mu \mathrm{m}$, and $100 \mu \mathrm{m}$ respectively is considered as the flow path for the continuous phase. The side channel of dimensions $100 \mu m \times 100 \mu m \times 100 \mu m$ was used for injecting the dispersed phase. A 1:5000 (by mass) solution of surfactant (Tween 80, Merck)-deionised water was used as the continuous phase and a light hydrocarbon oil-based ferrofluid EFH3 (Ferrotec) was used as the dispersed phase. The properties of the ferrofluid are given in Table 1. The surfactant was used to reduce the wetting of the microchannel by the ferrofluid. The VOF model implicitly assumes that the ferrofluid and the Tween-water solutions do not intermix, and a representative interfacial tension needs to be imposed. Although the surface tension of EFH3 ferrofluid and Tween-water solutions are available in literature, no reliable data about the interfacial tension between the two was found. Surfactant molecules from both the oil-based ferrofluid and the aqueous host fluid would absorb on the interface, altering the interfacial tension [41] to different extents, depending upon the nature of the exact surfactant used in the ferrofluid (surfactant data for EFH3 is not available since it is a commercially licensed product). To account for these uncertainties, therefore, the interfacial tension was determined in house experimentally (by the du Noüy ring method [42] at the interface of the same ferrofluid (EFH3) and the surfactant (Tween 80)-water solution considered in the simulation), and was found to be 0.01 $\mathrm{N} / \mathrm{m}$. The magnetic dipole was placed as shown in Fig. 2. Two positions of the dipole are shown, namely, upstream and downstream. For any simulation, only one dipole - either the upstream or the downstream - was active. Due to considerable distance between the magnet and the microfluidic channel, the magnet was modelled as a point dipole.

The dispersed phase entered through the side channel along the negative y-direction at a constant flow rate of $50 \mu \mathrm{L} / \mathrm{hr}$, while the velocity of the continuous phase (along the positive x-axis) was governed by the capillary number, $\mathrm{Ca}$. Plug velocity profiles were prescribed at both the inlets. The microchannel exit was modelled as a pressure outlet condition at atmospheric pressure. This mimics a typical microfluidic device where the channel terminates into a microfluidic well. No-slip boundary condition was specified at the channel walls. 


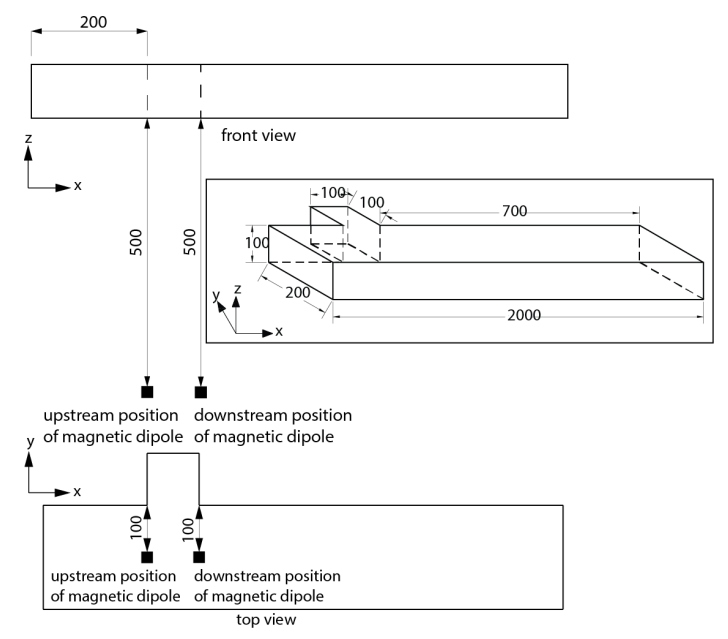

Figure 2: Position of the magnet with respect to the T-junction; (inset) Three-dimensional geometry of the Tjunction (not drawn to scale); upstream and downstream locations of the magnetic dipole shown (All dimensions are in $\mu \mathrm{m}$ )

\section{Governing Equations}

Both the dispersed (ferrofluid) and the continuous (surfacted liquid) phases were assumed to be incompressible, Newtonian in the present work, with the flow being isothermal, laminar. The Volume of Fluid (VOF) model was used in the present work to perform transient three dimensional simulations. The VOF method has been shown to be useful for simulating diverse problems such as evaporative cooling [43], pipeline corrosion [44], heat transfer in oscillating heat pipes [45], interaction of waves with coastal structures [46], liquid dessicant dehumidification [47], drop impact [48], and flow boiling [49] among others. The specialty of the VOF model is that it can model two or more immiscible fluids by solving a single set of momentum equations and tracking the volume fraction through the entire flow domain. A detailed description of the solution methods used in the present work is provided in Section 4.

The conservation equations for mass and momentum are expressed as

$$
\frac{\partial \rho}{\partial t}+\nabla \cdot(\rho \vec{V})=0
$$

and

$$
\rho\left(\frac{\partial \vec{V}}{\partial t}+\vec{V} \cdot \nabla \vec{V}\right)=-\nabla p+\nabla \cdot\left[\mu\left(\nabla \vec{V}+\nabla \vec{V}^{T}\right)\right]+\vec{F}_{s}+f \overrightarrow{F_{m}}
$$

respectively. For a two-phase system, the volume fraction of a cell, $f$, signifies the fraction of the total volume of the cell that is occupied by the dispersed phase. Therefore, $f=1$ indicates dispersed phase and $f=0$ indicates continuous phase, while a fractional value of $f$ signifies the interface. The transport equation for the volume fraction, $f$, is solved in the VOF method, which can be written as

$$
\frac{\partial f}{\partial t}+\vec{V} \cdot \nabla f=0
$$

Quantities $\rho$ and $\mu$ used in the above equations are the volume averaged density and viscosity, which are expressed as

$$
\begin{aligned}
& \rho=\rho_{1}+f\left(\rho_{2}-\rho_{1}\right) \\
& \mu=\mu_{1}+f\left(\mu_{2}-\mu_{1}\right)
\end{aligned}
$$

Compared to the other prevalent forces in microfluidic flow, gravitational force is not significant (since Bond number is small) and hence is neglected. The $\vec{F}_{s}$ term in the momentum equation is the surface tension force modelled using the Continuum Surface Force (CSF) formulation proposed by Brackbill et al. [50]. The surface force is expressed as

$$
\vec{F}_{s}=\sigma \frac{\rho \kappa \nabla f}{\frac{1}{2}\left(\rho_{1}+\rho_{2}\right)}
$$

The curvature of the interface, $\kappa$, is given by

$$
\kappa=-(\nabla \cdot \hat{n})
$$

where $\hat{n}$ is the unit normal vector given by

$$
\hat{n}=\frac{\nabla f}{|\nabla f|}
$$


Table 2: Mesh statistics

\begin{tabular}{ll}
\hline Nodes & 681226 \\
Elements & 640625 \\
Minimum orthogonal quality & 0.93 \\
Maximum aspect ratio & 2.75 \\
Element size & $4 \mu \mathrm{m}$ \\
\hline
\end{tabular}

In the present work, contact angle hysteresis and the effect of magnetic field on the contact angle were not considered. For ferrofluid droplets dispensed onto the substrate from the top, the left and right static contact angles were experimentally measured [51] to be $120^{\circ}$ and $125^{\circ}$ respectively. Hence, in the present work, a mean contact angle of $122.5^{\circ}$ was specified initially.

The $\overrightarrow{F_{m}}$ term in Eqn. 2 is the magnetic Kelvin Body Force (KBF). It is multiplied by the volume fraction, $f$, since it is only applicable for the ferrofluid (dispersed phase). For a typical two-particle system, the magnetic field, $\vec{H}$, is given by [52]

$$
\vec{H}=-\frac{\vec{m}}{r^{3}}+3(\vec{m} \cdot \vec{r}) \frac{\vec{r}}{r^{5}}
$$

The magnetization, $\vec{M}$, induced in a magnetic particle placed in a magnetic field, $\vec{H}$, can be written as

$$
\vec{M}=\chi \vec{H}
$$

where the magnetic susceptibilty, $\chi$, is a function of $\vec{H}$. From Maxwell's equations, in the absence of free electric currents (since the medium is nonmagnetic), it can be stated that [53]

$$
\nabla \times \vec{H}=0
$$

A magnetic particle in a nonmagnetic medium in the presence of a non-uniform magnetic field experiences a volumetric body force, termed as Kelvin Body Force (KBF), which can be expressed as [53]

$$
\overrightarrow{F_{m}}=\mu_{0}(\vec{M} \cdot \nabla) \vec{H}
$$

Using Eqns. 10, 11, and 12, the final expression of $\overrightarrow{F_{m}}$ turns out to be

$$
\overrightarrow{F_{m}}=\frac{1}{2} \mu_{0} \chi \nabla(\vec{H} \cdot \vec{H})
$$

The variation of $\chi$ with $H$ was determined experimentally [54], and a sixth order polynomial fit was used to approximate the nature of the variation. It is to be noted that in the present work, the 'non-inductive approximation' was considered in the magnetic field formulation, since the solid volume fraction in the ferrofluid was too weak to alter the imposed magnetic field [29]. Therefore, the estimation of magnetic field did not warrant a solution of the entire Maxwell's equations.

\section{Solution Method}

Numerical simulations were performed with the commercial finite volume method based CFD package ANSYS Fluent (v 14.5) using the unsteady segregated solver. The Pressure Implicit with Splitting of Operators (PISO) scheme was used for the pressure-velocity coupling, while the third-order Monotonic Upstream-Centered Scheme for Conservation Laws (MUSCL) scheme was used for the momentum equation. The pressure term was solved using the PREssure STaggering Option (PRESTO!) method. The Piecewise-Linear Interface Calculation (PLIC) technique [55] was used for interface reconstruction, and the transient term was treated using a first order implicit scheme. The convergence criteria for the continuity and momentum equations were set at $10^{-8}$. The ANSYS meshing package was used to create a quadrilateral based three-dimensional structured mesh. The time step size used was $10^{-4} s$, and the details of the computational mesh used is presented in Table 2. This optimum mesh was chosen after a rigorous grid independence study. This optimum mesh was chosen after a rigorous grid independence study. The run time for a typical simulation of 1 seconds flow time on a 20GB RAM computer having Intel-i7 processor was approximately 60 hours.

\section{$5 \quad$ Results and Discussions}

\subsection{Model validation}

For validation of the numerical model with the results of Sivasamy et al. [34], a commensurate domain length of $1000 \mu m$ was chosen (other dimensions as in Fig. 2). The gravitational force was considered while solving the 


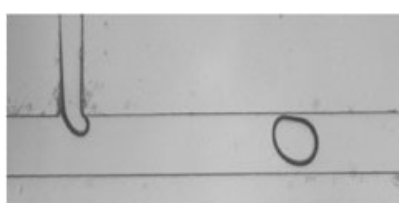

(a1) $\frac{t}{\tau}=0.5854$

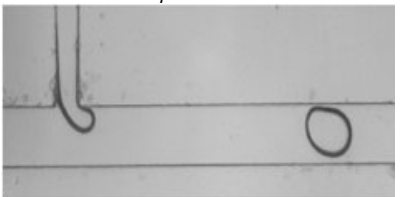

(b1) $\frac{t}{\tau}=0.8293$

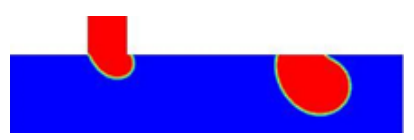

(a2) $\frac{t}{\tau}=0.6027$

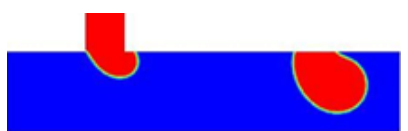

(b2) $\frac{t}{\tau}=0.8317$

Figure 3: Comparison of snapshots showing droplet formation at corresponding dimensionless time instants for $C a=0.025$; (a1), (b1) - experimental results reported by Sivasamy et al. [34]; (a2), (b2) - present simulations

momentum equation. The work of Sivasamy et al. [34] was chosen for validation since all relevant fluid properties used for their experiments were available in detail. In the present simulation, these fluid properties were used, along with the contact angle of the dispersed phase with the channel wall, and the continuous phase capillary number was fixed at 0.025. A comparison of the snapshots showing droplet formation in the experiments [34] with those of the present simulations at two different dimensionless time instants (time, $t$, non-dimensionalized with cycle time, i.e., time between two consecutive droplet detachments, $\tau$ ) is shown in Fig. 3. The comparison shows good agreement.

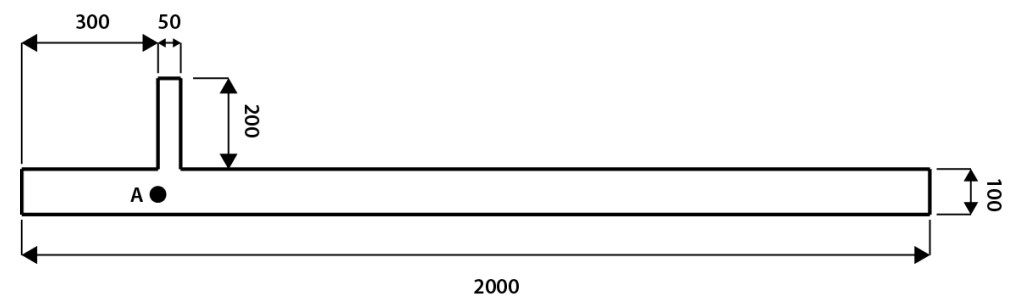

(a)

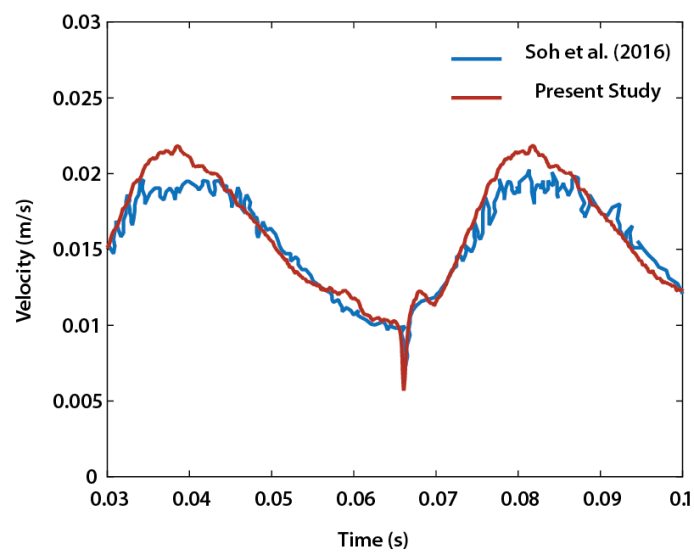

(b)

Figure 4: (a) T-junction geometry used for model validation (all dimensions are in $\mu \mathrm{m}$ ), the depth of the microchannel is $33 \mu \mathrm{m}$, A shows the location of the velocity probe; (b) comparison of velocity data recorded by the probe at $\mathrm{A}$ in the present work and as reported by Soh et al. [56]

A further quantitative validation of our numerical model was attempted against the work of Soh et al. [56]. The geometry chosen for this purpose was identical to the one used by Soh et al. [56] (Fig. 4(a)), while the velocity was recorded by a probe located at position A in Fig. 4(a). The continuous phase capillary number was maintained at 0.00232 and the dispersed phase flow rate was kept at $0.01 \mu \mathrm{L} / \mathrm{s}$. A comparison of the velocity history measured at probe position A in the present work that was reported by Soh et al. [56] is shown in Fig. 4(b), which shows excellent agreement. This clearly suggests that the numerical model used in the present work provides an accurate representation of the actual flow phenomenon. 

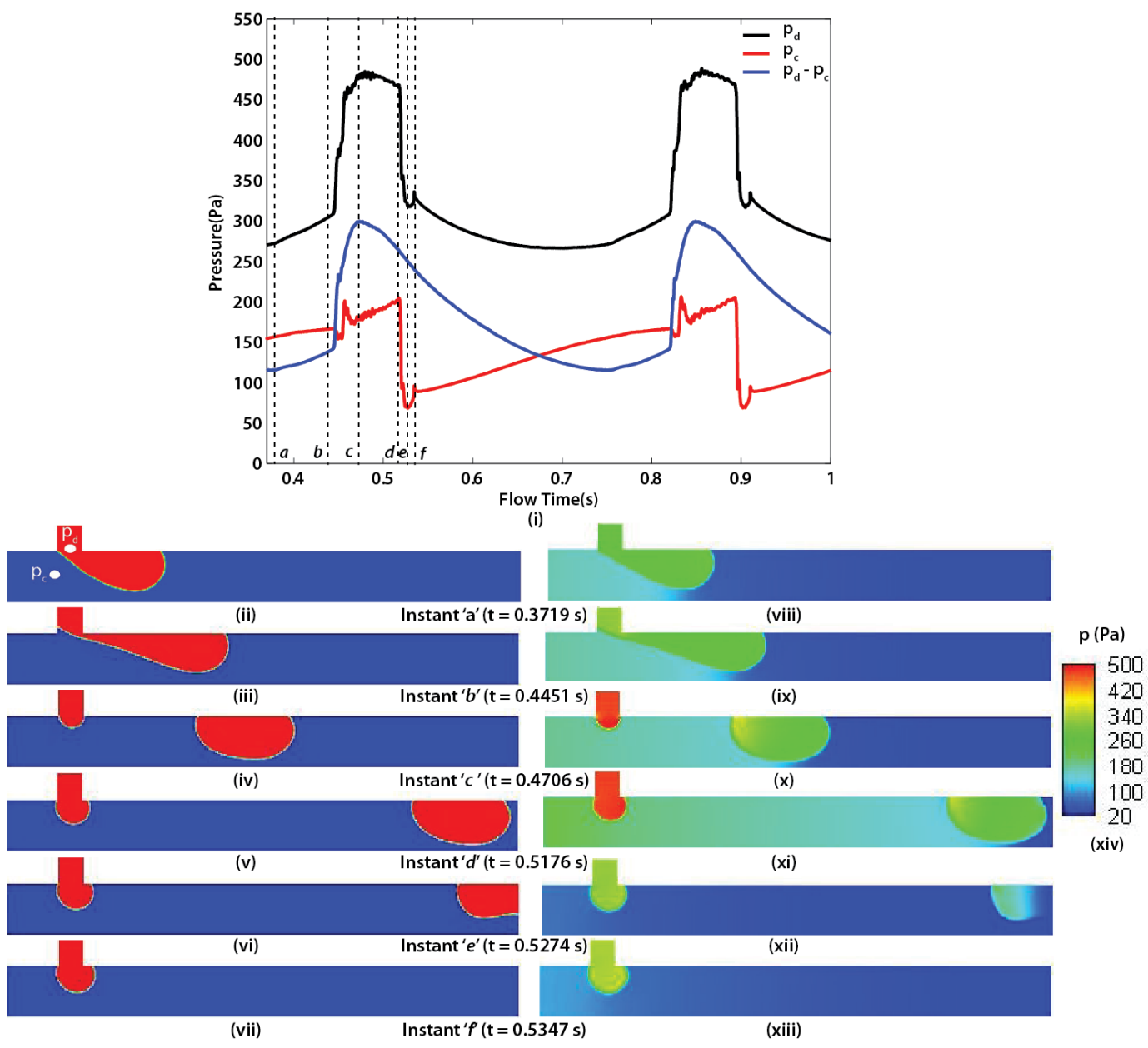

Figure 5: (i) Variation of continuous and dispersed phase pressures with flow time at $\mathrm{Ca}=0.025$ and $\mathrm{m}=0$; (ii) - (vii) droplet shapes at the salient time instants shown in (i); locations of the pressure probes are shown in inset (ii); (viii) - (xiii) pressure contours at the same salient time instants

\subsection{Ferrofluid flow in the absence of magnetic field}

For the present study, a continuous phase capillary number of 0.025 was considered as the base case. The variation of the dispersed and continuous phase pressures and their difference with flow time is shown in Fig. 5(i) for $\mathrm{Ca}=0.025$ and in the absence of any magnetic dipole (see Supplementary Material 1). The pressure plot is shown for two consecutive cycles of droplet generation. Droplet shapes (described in terms of the $f$ contour at the $x-y$ plane of symmetry through the channel) at a few salient time instants ' $a$ ' through ' $f$ ' are shown in Figs. 5(ii) through (vii), while the corresponding pressure contours at the geometric plane are depicted in Figs. 5(viii) through (xiii). When the ferrofluid thread enters the main channel from the side channel, the forces acting on it can be primarily attributed to the viscous, pressure, and surface stresses. From $a$ to $b$, the ferrofluid thread increases in volume, all the while remaining attached at the neck. The convex arc of the neck, however, slowly morphs into a concave shape as the tip of the thread penetrates further into the main channel. The pressure $p_{d}$ increases in this regime due to the change in curvature of the ferrofluid front. At the same time, $p_{c}$ increases as the increasing volume of the dispersed phase in the main channel reduces the flow area for the continuous phase (see Fig. 5(ii)), causing larger pressure drop in the continuous phase (as evident from the pressure drop past the droplet in Fig. 5(viii)). From $b$ to $c$ in Fig. $5, p_{d}$ sharply rises, which causes the increase in $p_{d}-p_{c}$. This pressure rise continues upto the point where the thread reaches the maximum volume, and the squeezing and shearing action of the continuous phase ruptures the neck, thus causing droplet detachment, followed by surface area minimisation of the droplet. As the detached droplet is advected downstream, it leaves the domain (instant $e$ ); the flow resistance offered by the droplet immediately disappears, causing a sudden drop in both $p_{d}$ and $p_{c}$. At the same time (starting from instant $d$ ) a fresh thread of ferrofluid enters from the side channel; as more volume of ferrofluid enters the main channel, curvature of the ferrofluid front decreases. This explains the steady descent in $p_{d}-p_{c}$ from instant $c$ to $d$. Beyond $f$, the dispersed phase slowly starts to push itself into the continuous phase, which causes $p_{d}$ to gradually decrease, and $p_{c}$ to gradually increase. The pressure cycle is found to repeat as the flow continues with a time period of $0.3762 \mathrm{~s}$ (evident from Fig. 5(i)). 


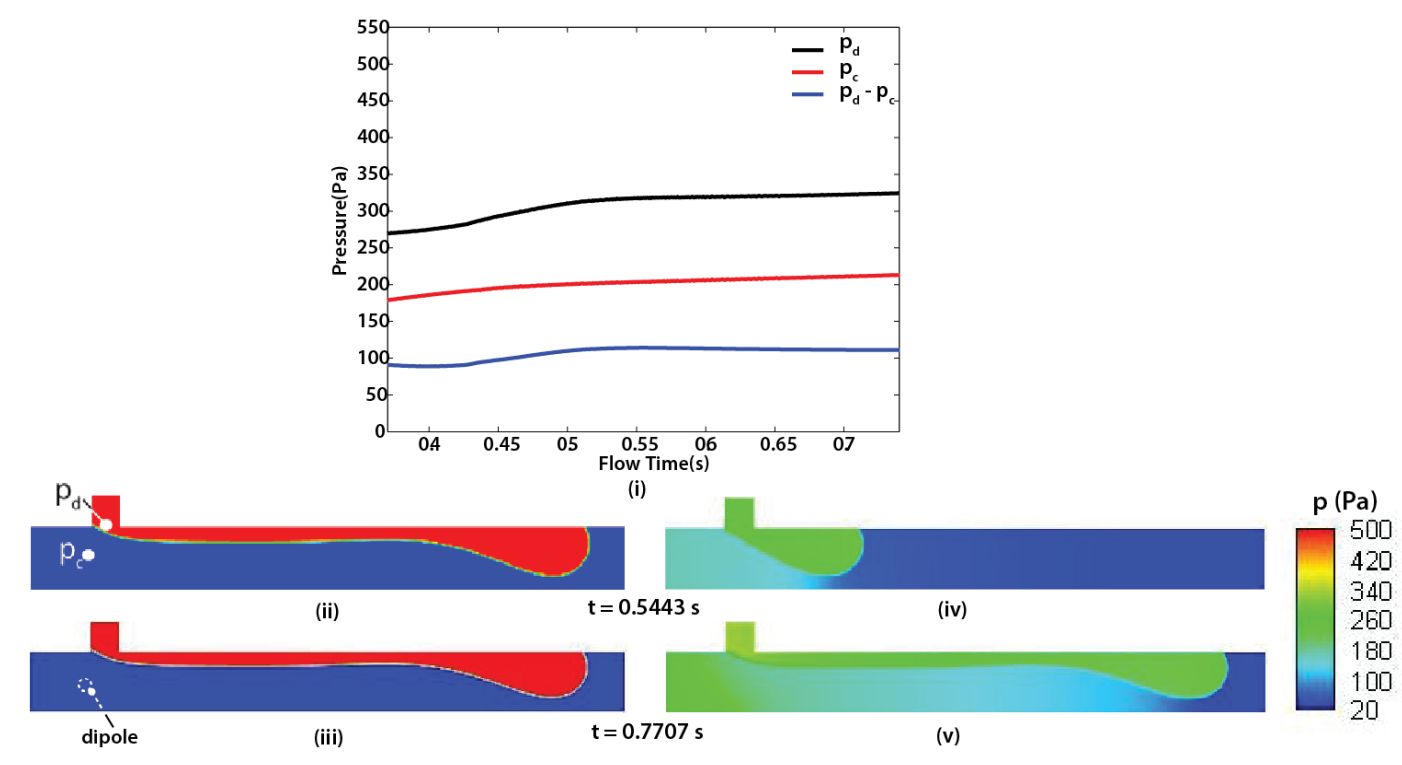

Figure 6: (i) Variation of continuous and dispersed phase pressures with flow time at $\mathrm{Ca}=0.025$ and $\mathrm{m}=$ $22.5 \times 10^{-6}$ A.m ${ }^{2}$ placed upstream; shapes of the dispersed phase at (ii) $t=0.5443 \mathrm{~s}$ and (iii) $t=0.7707 \mathrm{~s}$. Top views also show the locations of the pressure probes (inset (ii)) and location of the dipole under the microchannel (inset (iii)); (iv), (v) pressure contours at the same salient time instants

\subsection{Ferrofluid flow in the presence of magnetic dipole placed upstream}

A significant change in the ferrofluid flow behaviour (at the same $\mathrm{Ca}=0.025$ ) in the main channel is observed when a magnetic dipole of strength $22.5 \times 10^{-6} \mathrm{~A} . \mathrm{m}^{2}$ is placed at the upstream location. Droplet formation is completely suppressed; instead the ferrofluid flows through the main channel as a continuous stream parallel to the continuous phase (see Fig. 6 and Supplementary Material 2). This type of flow in literature is known as the jetting regime or parallel flow regime, as shown in Fig. 1(c). Traditionally, jetting occurs at high capillary numbers, where the shearing action of the continuous phase is so strong that it causes severe stretching of the dispersed phase in the main channel. In the present situation, in the presence of a magnetic dipole, a magnetic Kelvin body force also acts on the ferrofluid, in addition to the pressure, interfacial, and shear forces. The upstream placement of the dipole causes the ferrofluid to be attracted towards the dipole, thus opposing the direction of motion. This in turn reduces the dispersed phase velocity, thus increasing the relative velocity and hence the shear stress between the dispersed phase and the continuous phase. This increased shear stress is sufficient to take the system beyond the dripping-jetting transition point, thus enabling jetting to occur at a capillary number which is otherwise present in the dripping regime. Characteristic of the parallel flow regime, $p_{d}$ and $p_{c}$ also remain constant in this case. The steady $p_{d}-p_{c}$ value in Fig. 6 arises from the nearly unchanged Laplace pressure difference due to the curvature of the ferrofluid-water interface of the jet.

\subsection{Ferrofluid flow in the presence of magnetic dipole placed downstream}

When the same magnetic dipole of strength $22.5 \times 10^{-6} \mathrm{~A} \cdot \mathrm{m}^{2}$ is placed at the downstream location at the same $C a=0.025$, the flow behaviour (Fig. 7 for the pressure plots and the snapshots of several key time stamps, Supplementary Material 3) resembles that of the non-magnetic case (Fig. 5). In this case, as the ferrofluid flows through the side channel, the dipole is in its downstream direction, and thus the ferrofluid is attracted towards it. On entering the main channel, the tip of the ferrofluid penetrates into the continuous phase in the direction of the flow. But once it crosses the location of the magnetic dipole, the force reverses its direction, thereby impeding its forward flow. A reduction in the ferrofluid velocity occurs, thus increasing the velocity difference of the continuous and the dispersed phases, and increasing the shear stress exerted on ferrofluid by the continuous phase. However, this increase in shear stress is not as high as when the magnetic dipole was placed in the upstream position. Moreover, only the leading part of the ferrofluid thread experiences a magnetic force opposing the motion, while the trailing part still experiences the magnetic force in the direction of motion. Hence, the resulting shear stress on the ferrofluid is not sufficient to cross the dripping-jetting transition. However, since the attached ferrofluid thread and the detached droplet experiences a force in a direction against the flow, their residence time within the channel increases. Qualitatively, the pressure curves and the phenomena occurring at the points $a-f$ in Fig. 7 are similar to the non-magnetic case. However, the increased residence of the ferrofluid in the channel causes an increase in the duration for which the continuous phase flow path is blocked, hence increasing $p_{c}$ as compared to Fig. 5. As the $p_{d}$ remains unchanged, $p_{d}-p_{c}$ assumes much lower values compared 

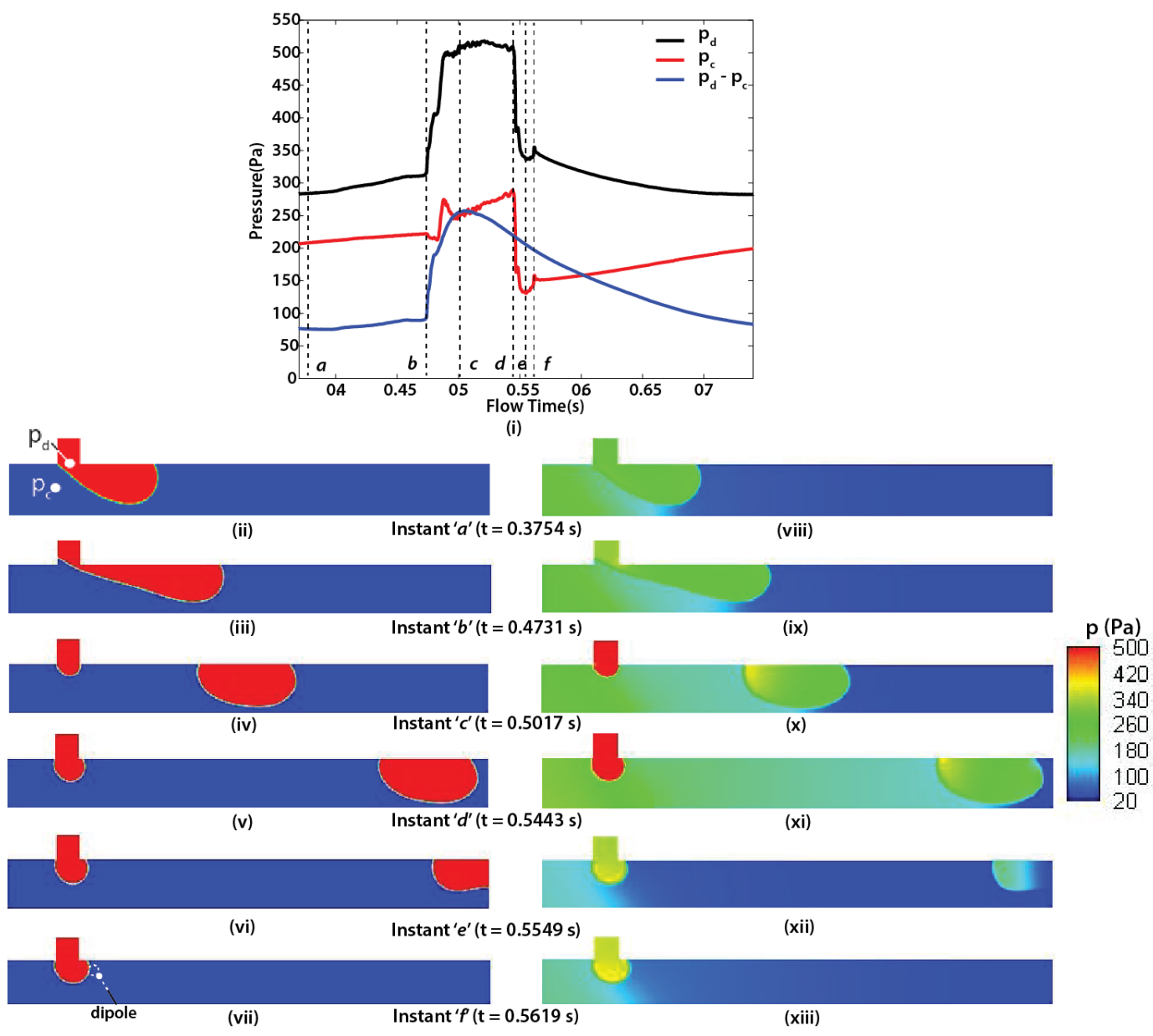

Figure 7: (i) Variation of continuous and dispersed phase pressures with flow time at $\mathrm{Ca}=0.025$ and $\mathrm{m}=$ $22.5 \times 10^{-6}$ A.m ${ }^{2}$ placed downstream; (ii) - (vii) droplet shapes at the salient time instants shown in (i); locations of the pressure probes are shown in inset (ii); (vii) location of the dipole under the microchannel; (viii) - (xiii) pressure contours at the same salient time instants

to the configuration corresponding to Fig. 5.

\subsection{Effect of dipole strength}

Figure 8 presents the variation of $p_{d}-p_{c}$ with flow time at $C a=0.025$ for different dipole strengths and for both upstream (Fig. 8(a)) and downstream (Fig. 8(b)) placements of the dipole. For both positions of the dipole,

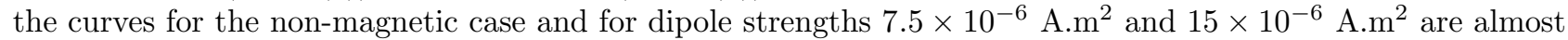
coincident. It is important to note here that the plots for the nonmagnetic case and for $m=7.5 \times 10^{-6} \mathrm{~A} . \mathrm{m}^{2}$ are nearly coincident, hence not distinguishable in Fig. 8(a). Like in the case reported in Fig. 5, different regimes of flow of the dispersed phase, comprising of penetration, squeezing, shearing, and detachment are observed in both the cases. This indicates that the magnetic force resulting from dipoles of such strengths are unable to influence the droplet formation procedure in the T-junction, and also points to the existence of a threshold dipole strength above which the magnetic force becomes significant. For upstream placement of the dipole, as observed in Fig. 6 , for $m=22.5 \times 10^{-6} \mathrm{~A} . \mathrm{m}^{2}$, droplet formation is suppressed and parallel flow is observed. On increasing the dipole strength to $26 \times 10^{-6} \mathrm{~A} . \mathrm{m}^{2}$, the parallel flow regime, expectedly, continues (since a higher dipole strength increases the magnetic force thus increasing the shear stress between the dispersed phase and the continuous phase). However, at this dipole strength, the $p_{d}-p_{c}$ curves assumes lower values than at $m=22.5 \times 10^{-6} \mathrm{~A} . \mathrm{m}^{2}$. This can be attributed to the "pulling effect" of the dipole on the side channel. For the downstream placement of the dipole, as observed in Fig. 7, droplet formation is not suppressed at $m=22.5 \times 10^{-6}$ A.m $^{2}$. However, the instant of droplet detachment (marked by ' $\mathrm{X}$ ' in Fig. 8(b)) is delayed. This is because of the increase in residence time of the droplet in the channel due to the increased attractive magnetic force felt by the droplet. An increase in $p_{c}$ due to increased residence time of the droplet (leading to increased duration of blockage of the continuous flow path) results in lower $p_{d}-p_{c}$ values compared to those at lower $m$ values. When the dipole strength is further increased to $26 \times 10^{-6} \mathrm{~A} . \mathrm{m}^{2}$, the attractive magnetic force on the droplet increases, thus further increasing the residence time of the droplet. This causes a delay in the droplet detachment, and also an 


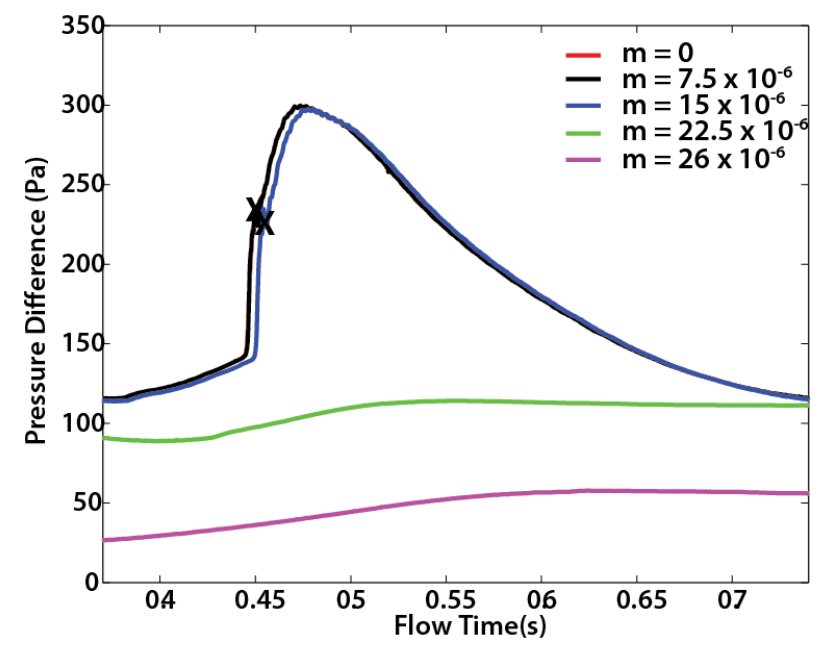

(a)

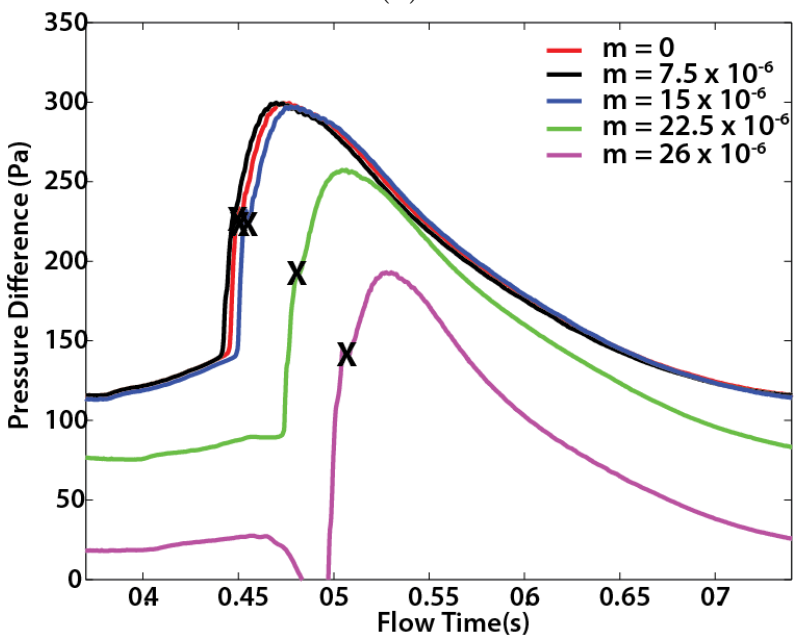

(b)

Figure 8: Variation of pressure difference between dispersed and continuous phases with flow time at $\mathrm{Ca}=0.025$ for different dipole strengths (in A. $\mathrm{m}^{2}$ ) for (a) upstream and (b) downstream placement of dipole; ' $\mathrm{X}$ ' marks the instant of droplet detachment. Pressure plots for $\mathrm{m}=0$ and $\mathrm{m}=7.5 \times 10^{-6}$ are almost identical at the upstream location, while those at the downstream location match closely.

increase in $p_{c}$, thus reducing $p_{d}-p_{c}$ to values lower than that at $m=22.5 \times 10^{-6} \mathrm{~A} . \mathrm{m}^{2}$.

\subsection{Effect of dipole placement}

The variation of $p_{d}-p_{c}$ with flow time at different $m$ values for both upstream and downstream placement of magnetic dipole is presented for $C a=0.025$ (Fig. 9(a)) and $C a=0.04$ (Fig. 9(b)). Since the magnetic force at $m=15 \times 10^{-6} \mathrm{~A} . \mathrm{m}^{2}$ is not strong enough to influence the droplet formation procedure (as observed from Fig. 8), the $p_{d}-p_{c}$ curves at that value for both upstream and downstream placement of the dipole appear coincident for both $C a=0.025$ and $C a=0.04$ cases. Fig. 9(a) essentially represents Fig. 8(a) and Fig. 8(b) in a single frame, and hence is not elaborated upon again for the sake of brevity. Figure 9(b) shows that, unlike the $C a=0.025$ case, droplet formation still occurs at $m=22.5 \times 10^{-6} \mathrm{~A} \cdot \mathrm{m}^{2}$ for $C a=0.04$, and it is suppressed only at $m=26 \times 10^{-6} \mathrm{~A} \cdot \mathrm{m}^{2}$. As $C a$ is increased, the continuous phase velocity increases, which increases the shear stress on the dispersed phase. Hence, the droplet tends to be detached much quicker, but an attractive magnetic force opposes it. However, this also means that a larger dipole strength would be required to tip the system past the dripping-jetting transition point. This causes the appearance of jetting at $C a=0.04$ at higher $m$ values as compared to $C a=0.025$. Comparing the $p_{d}-p_{c}$ curves at $m=22.5 \times 10^{-6} \mathrm{~A}^{-\mathrm{m}^{2}}$ for $C a=0.04$ for both upstream and downstream positions of the dipole, it is observed that droplet detachment (given by the peak in the $p_{d}-p_{c}$ curve) occurs at a much earlier time instant for a dipole in the downstream direction (detachment occurs at $t=0.3851 \mathrm{~s}$ ) rather than that placed at the upstream direction (detachment occurs at $t=0.4099 \mathrm{~s}$ ). This delayed detachment can be attributed to the fact that a downstream dipole is not able to slow down the ferrofluid as much as the upstream dipole. With the downstream position of the dipole, the leading part of the 


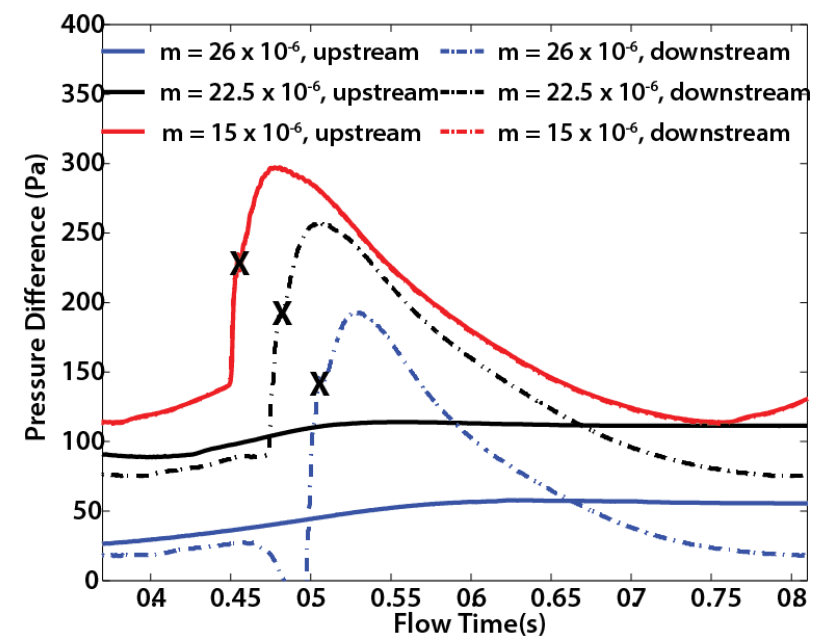

(a)

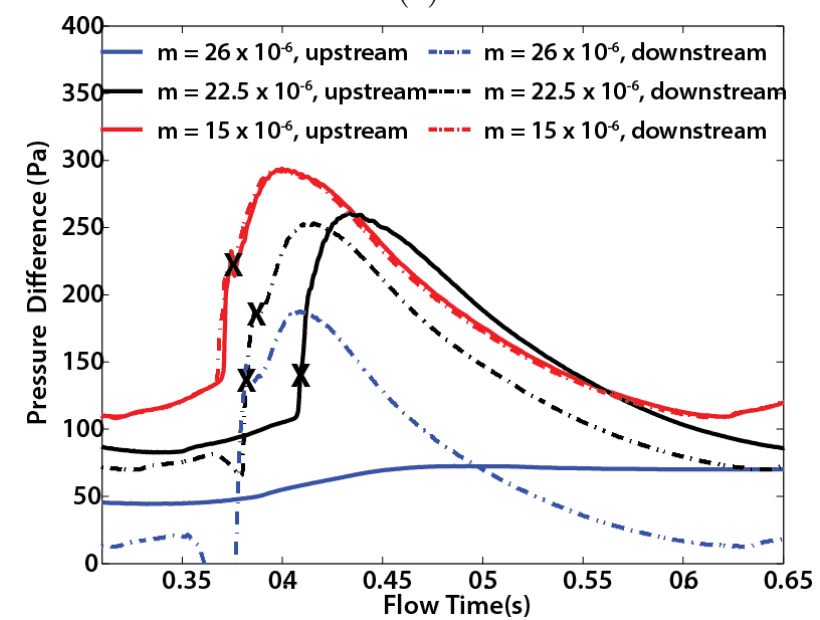

(b)

Figure 9: Variation of pressure difference between dispersed and continuous phases with flow time for both upstream and downstream positions of magnetic dipole of different strengths (in A.m ${ }^{2}$ ) at (a) $\mathrm{Ca}=0.025$ and (b) $\mathrm{Ca}=0.04$; ' $\mathrm{X}$ ' marks the instant of droplet detachment

ferrofluid thread experiences magnetic force opposite to the flow direction while the trailing part experiences magnetic force in the direction of motion. On the contrary the entire ferrofluid thread experiences magnetic force opposite to the direction of motion in the upstream dipole case, thus reducing the residence time in the downstream dipole case as compared to the upstream dipole. This causes the delayed droplet detachment time in the upstream dipole case compared to the downstream dipole case for $m=22.5 \times 10^{-6}$ A.m ${ }^{2}$ at $C a=0.04$. When the dipole strength is increased to $26 \times 10^{-6} \mathrm{~A} \cdot \mathrm{m}^{2}$ at $C a=0.04$, the upstream dipole case results in jetting due to the increase in shear stresses to values beyond the jetting-dripping transition, whereas the downstream dipole case still results in droplets. It is interesting to note that the droplet detachment time instants are almost same for both $m=22.5 \times 10^{-6} \mathrm{~A} \cdot \mathrm{m}^{2}$ and $m=26 \times 10^{-6} \mathrm{~A} \cdot \mathrm{m}^{2}$ in the downstream dipole case. The $p_{d}-p_{c}$ values at $m=26 \times 10^{-6} \mathrm{~A} . \mathrm{m}^{2}$ are expectedly less than those corresponding to $m=22.5 \times 10^{-6}$ A.m $\mathrm{m}^{2}$ in the downstream dipole case because of higher values of $p_{c}$ caused by the increase in residence time of a droplet due to a dipole of greater strength.

\subsection{Effect of capillary number}

The effect of changing $C a$ from 0.025 to 0.04 on the $p_{d}-p_{c}$ curve is shown in Fig. 10 for both upstream (Fig. 10(a)) and downstream (Fig. 10(b)) placement of the magnetic dipole, and at three different dipole strengths. Increase in $\mathrm{Ca}$ basically means that the velocity of the continuous phase increases, thus increasing the shear stress exerted on the dispersed phase. This promotes droplet breakup in the dripping regime, and expectedly the droplet formation frequency increases (as observed in Fig. 10). The $p_{d}-p_{c}$ curves for $C a=0.04$ leads the $p_{d}-p_{c}$ curve for $C a=0.025$ in both the upstream and downstream dipole cases. The peak values of $p_{d}-p_{c}$ reached for each of the droplet formation cases is independent of $C a$ and only depends on the $m$ value. For example, the peak values reached for $m=15 \times 10^{-6} \mathrm{~A} \cdot \mathrm{m}^{2}$ is same in both the upstream and downstream dipole 


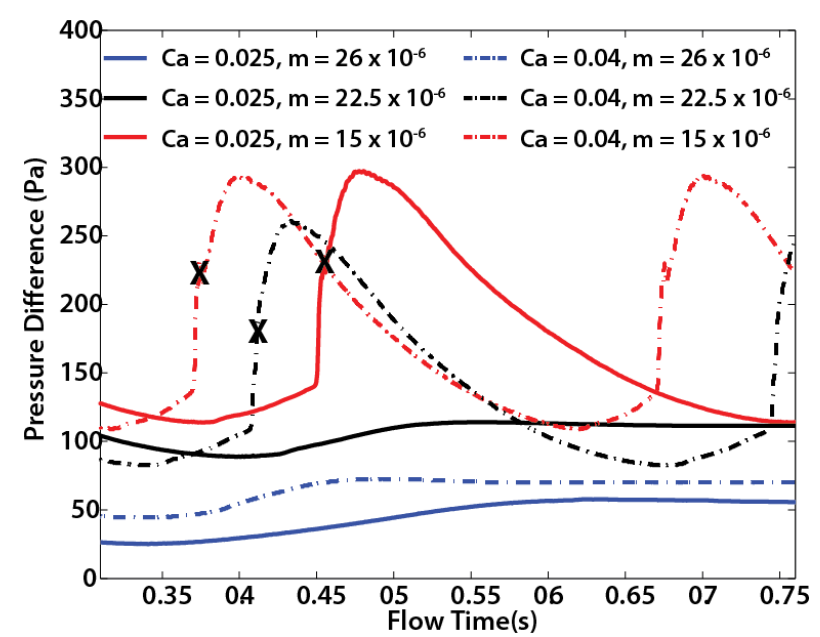

(a)

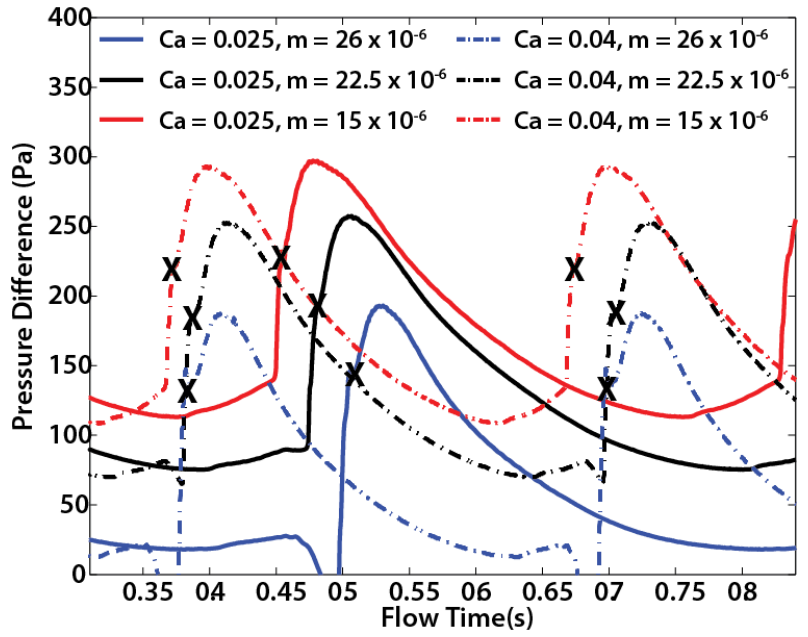

(b)

Figure 10: Variation of pressure difference between dispersed and continuous phases with flow time at $\mathrm{Ca}=$ 0.025 and $\mathrm{Ca}=0.04$ for different magnetic dipole strengths (in A. $\mathrm{m}^{2}$ ) for (a) upstream and (b) downstream placement of dipole; ' $\mathrm{X}$ ' marks the instant of droplet detachment

position cases, irrespective of the $C a$ value. The changes in the droplet formation time periods are also evident in the $p_{d}-p_{c}$ curves. The droplet formation time period is less for $C a=0.04$ cases than the corresponding $C a=0.025$ cases. However, for constant $C a$, the time period increases with increasing $m$. This can be attributed to the increase in residence time of the droplet in the channel for higher dipole strengths.

\subsection{Droplet characteristics}

An important characteristic of any digital microfluidic system is the frequency at which droplets are generated. Figure 11 presents the variation of droplet shedding period (cycle time) with dipole strength for different $\mathrm{Ca}$ values and dipole positions. A detailed explanation for the change in cycle time has already been provided in Section 5.7. In a digital microfluidic platform, the size of dispensed droplets is also reckoned as an important parameter. Therefore, we investigate the influence of the capillary number and the dipole strength and position on the droplet size. Respective droplet sizes for $C a=0.025$ and $C a=0.04$ for a downstream magnetic dipole of strength $22.5 \times 10^{-6} \mathrm{~A} . \mathrm{m}^{2}$ are shown in Figs. 12(a) and 12(b). For a fixed dispersed phase velocity, the droplet size decreases with increasing $C a$ due to increased shear stresses associated with increased continuous phase velocity. This is also evident in Fig. 12(a) and Fig. 12(b). The droplet size for $C a=0.04$ is evidently smaller than that of $C a=0.025$, and the former also blocks less of the channel area than the latter. To quantitatively characterize the droplet sizes, two linear dimensions $h$ and $b$ are introduced, as shown in Fig. 12(c). The variation of $h$ and $b$ with $C a$ and $m$ is shown in Table 3. It is evident from Table 3 that the $h$ and $b$ values for droplets at $C a=0.025$ are expectedly higher than the corresponding values at $C a=0.04$. Also, for each $C a$ value, the $h$ and $b$ values remain constant as the magnetic dipole strength is increased from 0 to $15 \times 10^{-6}$ A.m ${ }^{2}$. This is due to the fact that the magnetic forces resulting from these dipole strengths are not sufficient to alter the droplet formation procedure, as detailed in previous sections. The droplet size increases as $m$ is increased 


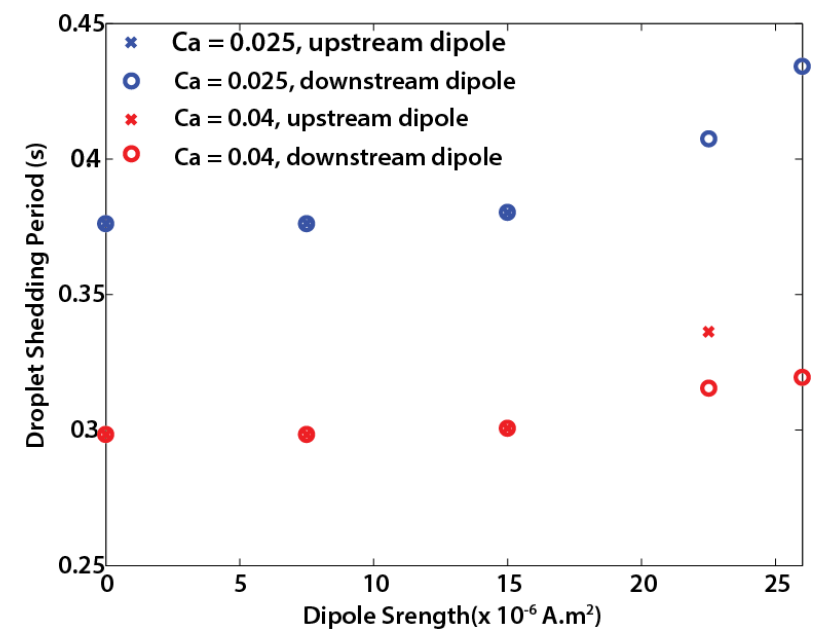

Figure 11: Variation of droplet shedding period with dipole strength for different $C a$ and dipole positions

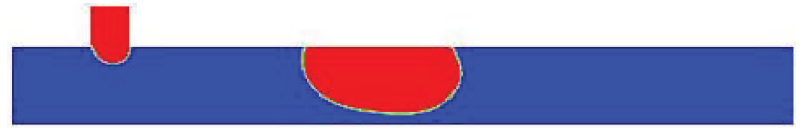

(a) $C a=0.025, m=22.5 \times 10^{-6} \mathrm{~A} . \mathrm{m}^{2}$, downstream

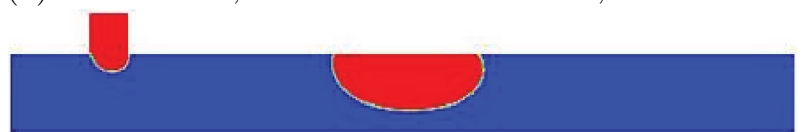

(b) $C a=0.04, m=22.5 \times 10^{-6} \mathrm{~A} . \mathrm{m}^{2}$, downstream

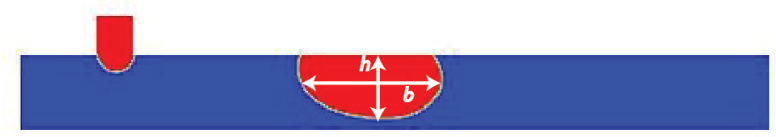

(c) Characteristic droplet dimensions $b$ and $h$

Figure 12: Representative droplet sizes at $C a=0.025$ and $C a=0.04$ for same magnetic dipole strength and position; characteristic droplet dimensions $h$ and $b$

beyond $15 \times 10^{-6}$ A.m ${ }^{2}$ for both $C a=0.025$ and $C a=0.04$, as evident from the increased $h$ and $b$ values. This is because of the increase in the time period of droplet formation, resulting in accumulation of a greater volume of ferrofluid within the thread prior to detachment. The droplet size increases as $m$ is increased from $22.5 \times 10^{-6}$ A.m ${ }^{2}$ to $26 \times 10^{-6}$ A.m ${ }^{2}$ for $C a=0.025$ due to the increased magnetic force associated with greater dipole strength. Similar trends are also observed for droplet sizes at $C a=0.04$. It is interesting to note that, at $C a=0.04$, the droplet size is greater for dipole of strength $22.5 \times 10^{-6} \mathrm{~A} . \mathrm{m}^{2}$ placed upstream than for dipole of strength $26 \times 10^{-6} \mathrm{~A} \cdot \mathrm{m}^{2}$ placed downstream. This is also apparent from Fig. 10(b), where the time period of droplet formation was less for $m=26 \times 10^{-6}$ A.m ${ }^{2}$ placed downstream than that for $m=22.5 \times 10^{-6}$ A.m ${ }^{2}$ placed upstream. This provides insight into the correlation between droplet size and shedding time period which is explained as follows using droplet volume. Moreover, droplet volumes are a good indicator of the throughput of a digital microfluidic system. A new parameter, $V_{e}$ is introduced, which is defined as:

$$
V_{e}=\frac{V-V_{0}}{V_{0}} \times 100
$$

where $V$ is the volume of the droplet for a particular $C a$ and $m$ value and a particular dipole position, and $V_{0}$ is the droplet volume for the nonmagnetic case at that corresponding $C a$. The variation of $V_{e}$ with $C a$ and $m$ is also shown in Table 3. High $V_{e}$ values observed at higher dipole strengths can be attributed to the higher droplet shedding time periods observed at those values. For a higher droplet shedding time, the droplet detachment is delayed, but the dispersed phase is continuously fed for that additional time, resulting in the increased volumes.

\section{Conclusion}

In the present work, three-dimensional VOF simulations of ferrofluid droplet generation in a microfluidic T-junction in presence of an external magnetic dipole were carried out. The velocity of the dispersed phase 
Table 3: Droplet dimensions and excess volumes at different $C a$ and $m$ values

\begin{tabular}{llllll}
\hline$C a$ & $m\left(\mu \mathrm{A} . \mathrm{m}^{2}\right)$ & Dipole Location & $b(\mu \mathrm{m})$ & $h(\mu \mathrm{m})$ & $V_{e}(\%)$ \\
\hline 0.025 & 0 & N/A & 389 & 170 & 0 \\
0.025 & 7.5 & Upstream & 389 & 170 & 0.03 \\
0.025 & 7.5 & Downstream & 389 & 170 & 0.03 \\
0.025 & 15 & Upstream & 389 & 170 & 1.06 \\
0.025 & 15 & Downstream & 389 & 170 & 1.09 \\
0.025 & 22.5 & Downstream & 410 & 173 & 8.29 \\
0.025 & 26 & Downstream & 428 & 173 & 15.44 \\
0.04 & 0 & N/A & 376 & 142 & 0 \\
0.04 & 7.5 & Upstream & 376 & 142 & 0 \\
0.04 & 7.5 & Downstream & 376 & 142 & 0 \\
0.04 & 15 & Upstream & 376 & 142 & 0.77 \\
0.04 & 15 & Downstream & 376 & 142 & 0.77 \\
0.04 & 22.5 & Upstream & 400 & 144 & 12.70 \\
0.04 & 22.5 & Downstream & 389 & 144 & 5.73 \\
0.04 & 26 & Downstream & 394 & 144 & 7.07 \\
\hline
\end{tabular}

(ferrofluid) was kept constant, while two different capillary numbers of the continuous phase, viz., 0.025 and 0.04, were considered. The magnetic dipole strength was varied from 0 (non-magnetic case) to $26 \times 10^{-6} \mathrm{~A} . \mathrm{m}^{2}$, and two distinct positions of the dipole were considered - upstream and downstream. A detailed report on the outcomes of such simulations is given in Table 4 . For the non-magnetic case $(m=0)$, both $C a=0.025$

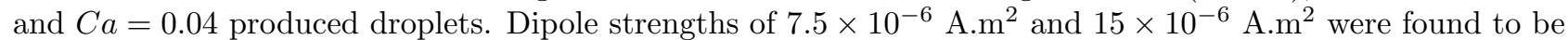
insufficient to alter the flow behaviour of the ferrofluid. Only for $m=22.5 \times 10^{-6} \mathrm{~A} . \mathrm{m}^{2}$ and $m=26 \times 10^{-6}$ A.m ${ }^{2}$, significant changes in the flow behaviour were observed. At $C a=0.025$, for a dipole of strength $22.5 \times 10^{-6}$ A. $\mathrm{m}^{2}$ placed at the upstream location, droplet formation was suppressed and parallel flow between the ferrofluid and the continuous phase was observed. Such a behaviour was also observed at an even higher dipole strength of $26 \times 10^{-6}$ A.m² . However, for downstream position of dipoles, droplet formation was never suppressed, which is contrary to the upstream position of the dipole of same strength. For $C a=0.04$, suppression of droplet formation

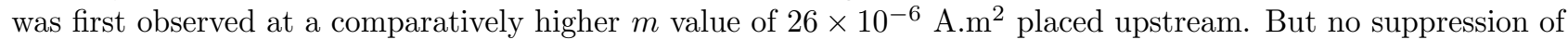
droplet formation was again observed at this capillary number for magnetic dipoles placed downstream. The droplet detachment time period was observed to increase with increasing $m$ once the magnetic force became significant enough to alter the flow behaviour. However, the droplet time period for an upstream dipole was greater than that for the same dipole placed downstream. The droplets at $C a=0.04$ were also expectedly smaller than the corresponding droplets at $C a=0.025$. However, for fixed $C a$, droplet size was observed to increase with increasing $m$ beyond the threshold $m$ value. Results of the study provide an insight into active control of droplet generation in a digital microfluidic platform and lends to design basis of microfluidic droplet generation devices. 
Table 4: Ferrofluid flow behaviour at different $C a$ and $m$ values

\begin{tabular}{llll}
\hline$C a$ & $m\left(\mu \mathrm{A} . \mathrm{m}^{2}\right)$ & Dipole Location & Droplet/ Jet \\
\hline 0.025 & 0 & N/A & Droplet \\
0.025 & 7.5 & Upstream & Droplet \\
0.025 & 7.5 & Downstream & Droplet \\
0.025 & 15 & Upstream & Droplet \\
0.025 & 15 & Downstream & Droplet \\
0.025 & 22.5 & Upstream & Jet \\
0.025 & 22.5 & Downstream & Droplet \\
0.025 & 26 & Upstream & Jet \\
0.025 & 26 & Downstream & Droplet \\
0.04 & 0 & N/A & Droplet \\
0.04 & 7.5 & Upstream & Droplet \\
0.04 & 7.5 & Downstream & Droplet \\
0.04 & 15 & Upstream & Droplet \\
0.04 & 15 & Downstream & Droplet \\
0.04 & 22.5 & Upstream & Droplet \\
0.04 & 22.5 & Downstream & Droplet \\
0.04 & 26 & Upstream & Jet \\
0.04 & 26 & Downstream & Droplet \\
\hline
\end{tabular}

\section{References}

[1] M. Kühnemund, D. Witters, M. Nilsson, and J. Lammertyn. Circle-to-circle amplification on a digital microfluidic chip for amplified single molecule detection. Lab Chip, 14:2983-2992, 2014.

[2] V. N. Luk and A. R. Wheeler. A digital microfluidic approach to proteomic sample processing. Anal. Chem., 81:4524-4530, 2009.

[3] N. A. Mousa, M .J. Jebrail, H. Yang, M. Abdelgawad, P. Metalnikov, J. Chen, A. R. Wheeler, and R. F. Casper. Droplet-scale Estrogen assays in breast tissue, blood, and serum. Sci. Transl. Med., 1(1):1-6, 2009.

[4] P. Y. Keng, S. Chen, H. Ding, S. Sadeghi, G. J. Shah, A. Dooraghi, M. E. Phelps, N. Satyamurthy, A. F. Chatziioannou, C.-J. Kim, and R. M. van Dam. Micro-chemical synthesis of molecular probes on an electronic microfluidic device. Proc. Natl. Acad. Sci., 109(3):690-695, 2012.

[5] R. S. Sista, A. E. Eckhardt, V. Srinivasan, M. G. Pollack, S. Palanki, and V. K. Pamula. Heterogeneous immunoassays using magnetic beads on a digital microfluidic platform. Lab Chip, 8:2188-2196, 2008.

[6] I. Barbulovic-Nad, H. Yang, P. S. Park, and A. R. Wheeler. Digital microfluidics for cell-based assays. Lab Chip, 8:519-526, 2008.

[7] Y.-H. Chang, G.-B. Lee, F.-C. Huang, Y.-Y. Chen, and J.-L. Lin. Integrated polymerase chain reaction chips utilizing digital microfluidics. Biomed. Microdevices, 8:215-225, 2006.

[8] R. B. Fair. Digital microfluidics: is a true lab-on-a-chip possible? Microfluid. Nanofluid., 3:245-281, 2007.

[9] M. J. Jebrail, M. S. Bartsch, and K. D. Patel. Digital microfluidics: a versatile tool for applications in chemistry, biology and medicine. Lab Chip, 12:2452-2463, 2012.

[10] K. Choi, A. H. C. Ng, R. Fobel, and A. R. Wheeler. Digital microfluidics. Annu. Rev. Anal. Chem., 5:413-440, 2012.

[11] C. Cramer, P. Fischer, and E. J. Windhab. Drop formation in a co-flowing ambient fluid. Chem. Eng. Sci., 59:3045-3058, 2004.

[12] M. Herrada, J. M. Montanero, C. Ferrera, and A. M. Gañán Calvo. Analysis of the dripping-jetting transition in compound capillary jets. J. Fluid Mech., 649:523-536, 2010.

[13] A. Perro, C. Nicolet, J. Angly, S. Lecommandoux, J.-F. Le Meins, and A. Colin. Mastering a double emulsion in a simple co-flow microfluidic to generate complex polymersomes. Langmuir, 27:9034-9042, 2010. 
[14] T. Ward, M. Faivre, M. Abkarian, and H. A. Stone. Microfluidic flow focusing:Drop size and scaling in pressure versus flow-rate-driven pumping. Electrophoresis, 26:3716-3724, 2005.

[15] P. Garstecki, H. A. Stone, and G. M. Whitesides. Mechanism for flow-rate controlled breakup in confined geometries: A route to monodisperse emulsions. Phys. Rev. Lett., 94:164501, 2005.

[16] A. S. Utada, E. Lorenceau, D. R. Link, P. D. Kaplan, H. A. Stone, and D. A. Weitz. Monodisperse double emulsions generated from a microcapillary device. Science, 308:537-541, 2005.

[17] D. N. Josephides and S. Sajjadi. Increased drop formation frequency via reduction of surfactant interactions in flow-focusing microfluidic devices. Langmuir, 31:1218-1224, 2015.

[18] P. Garstecki, M. J. Fuerstman, H. A. Stone, and G. M. Whitesides. Formation of droplets and bubbles in a microfluidic T-junction - scaling and mechanism of break-up. Lab Chip, 6:437-446, 2006.

[19] A. Gupta, S. M. S. Murshed, and R. Kumar. Droplet formation and stability of flows in a microfluidic T-junction. Appl. Phys. Lett., 94:164107, 2009.

[20] H. Liu and Y. Zhang. Droplet formation in a T-shaped microfluidic junction. J. Appl. Phys., 106:034906, 2009.

[21] V. van Steijn, C. R. Kleijn, and M. T. Kreutzer. Predictive model for the size of bubbles and droplets created in microfluidic T-junctions. Lab Chip, 10:2513-2518, 2010.

[22] M. G. Pollack, A. D. Shenderov, and R. B. Fair. Electrowetting-based actuation of droplets for integrated microfluidics. Lab Chip, 2:96-101, 2002.

[23] A. R. Wheeler. Putting electrowetting to work. Science, 332:539-540, 2008.

[24] G. J. Shah, A. T. Ohta, E. P.-Y. Chiou, M. C. Wu, and C.-J. Kim. EWOD-driven droplet microfluidic device integrated with optoelectronic tweezers as an automated platform for cellular isolation and analysis. Lab Chip, 9:1732-1739, 2009.

[25] S. K. Cho, Y. Zhao, and C.-J. Kim. Concentration and binary separation of micro particles for droplet-based digital microfluidics. Lab Chip, 7:490-498, 2007.

[26] H. R. Nejad, O. Z. Chowdhury, M. D. Buat, and M. Hoorfar. Characterization of the geometry of negative dielectrophoresis traps for particle immobilization in digital microfluidic platforms. Lab Chip, 13:1823-1830, 2013.

[27] Y. Wang, Y. Zhao, and S. K. Cho. Efficient in-droplet separation of magnetic particles for digital microfluidics. J. Micromech. Microeng., 17:2148-2156, 2007.

[28] Z. Long, A. M. Shetty, M. J. Solomon, and R. G. Larson. Fundamentals of magnet-actuated droplet manipulation on an open hydrophobic surface. Lab Chip, 9:1567-1575, 2009.

[29] R. E. Rosensweig. Ferrohydrodynamics. Dover Publications Inc., 1998.

[30] S. Odenbach. Recent progress in magnetic fluid research. J. Phys. Condens. Matter, 16:R1135-R1150, 204.

[31] N.-T. Nguyen. Micro-magnetofluidics: interactions between magnetism and fluid flow on the microscale. Microfluid. Nanofluid., 12:1-16, 2012.

[32] M. de Menech, P. Garstecki, F. Jousse, and H. A. Stone. Transition from squeezing to dripping in a microfluidic T-junction. J. Fluid Mech., 595:141-161, 2008.

[33] C.-Y. Chen, C.-H. Chen, and W.-F. Lee. Experiments on breakup of a magnetic fluid drop through a micro-orifice. J. Magn. Magn. Mater., 321:3520-3525, 2009.

[34] J. Sivasamy, T.-N. Wong, N.-T. Nguyen, and L. T.-H. Kao. An investigation on the mechanism of droplet formation in a microfluidic T-junction. Microfluid. Nanofluid., 11:1-10, 2011.

[35] S. H. Tan and N.-T. Nguyen. Generation and manipulation of monodispersed ferrofluid emulsions: The effect of a uniform magnetic field in flow-focusing and T-junction configurations. Phys. Rev. E, 84:036317, 2011.

[36] Y. Wu, T. Fu, Y. Ma, and H. Z. Li. Ferrofluid droplet formation and breakup dynamics in a microfluidic flow-focusing device. Soft Matter, 9:9792-9798, 2013. 
[37] Q. Yan, S. Xuan, X. Ruan, J. Wu, and X. Gong. Magnetically controllable generation of ferrofluid droplets. Microfluid. Nanofluid., 19:1377-1384, 2015.

[38] Y. Wu, T. Fu, Y. Ma, and H. Z. Li. Active control of ferrofluid droplet breakup dynamics in a microfluidic T-junction. Microfluid. Nanofluid., 18:19-27, 2015.

[39] J. Liu, Y. F. Yap, and N.-T. Nguyen. Numerical study of the formation process of ferrofluid droplets. Phys. Fluids, 23:072008, 2011.

[40] S.-H. Tan, N.-T. Nguyen, L. Yobas, and T. G. Kang. Formation and manipulation of ferrofluid droplets at a microfluidic T-junction. J. Micromech. Microeng., 20:045004, 2010.

[41] J.-C. Baret. Surfactants in droplet-based microfluidics. Lab Chip, 12:422-433, 2012.

[42] P.-G. de Gennes, F. Brochard-Wyart, and D. Quéré. Capillarity and Wetting Phenomena: Drops, Bubbles, Pearls, Waves. Springer-Verlag New York Inc., 2004.

[43] J. N. Chen, Z. Zhang, X. L. Ouyang, and P. X. Jiang. Dropwise evaporative cooling of heated surfaces with various wettability characteristics obtained by nanostructure modifications. Nanoscale Res. Lett., 11:158, 2016.

[44] H. Hu and Y. F. Cheng. Modeling by computational fluid dynamics simulation of pipeline corrosion in $\mathrm{CO}_{2}$-containing oil-water two phase flow. J. Petrol. Sci. Eng., 146:134-141, 2016.

[45] J. E, X. Zhao, H. Liu, J. Chen, W. Zuo, and Q. Peng. Field synergy analysis for enhancing heat transfer capability of a novel narrow-tube closed oscillating heat pipe. Appl. Energy, 175:218-228, 2016.

[46] X. Zhao, Y. Gao, F. Cao, and X. Wang. Numerical modeling of wave interactions with coastal structures by a constrained interpolation profile/immersed boundary method. Int. J. Numer. Meth. Fluids, 81:265-283, 2016.

[47] H. Lu, L. Lu, Y. Luo, and R. Qi. Investigation on the dynamic characteristics of the counter-current flow for liquid dessicant dehumidification. Energy, 101:229-238, 2016.

[48] J. B. Lee, D. Derome, A. Dolatabadi, and J. Carmeliet. Energy budget of liquid drop impact at maximum spreading: Numerical simulations and experiments. Langmuir, 32:1279-1288, 2016.

[49] Z. Pan, J. A. Weibel, and S. V. Garimella. A saturated-interface-volume phase change model for simulating flow boiling. Int. J. Heat Mass Transfer, 93:945-956, 2016.

[50] J. U. Brackbill, D. B. Kothe, and C. Zemach. A continuum method for modeling surface tension. J. Comput. Phys., 100:335-354, 1992.

[51] S. Chatterjee, D. Bhowmik, A. Mukhopadhyay, and R. Ganguly. Analysis of static and dynamic contact angles of ferrofluid droplets for magnetically actuated micropumps. In $41^{\text {st }}$ National and $5^{\text {th }}$ International Conference on Fluid Mechanics and Fluid Power, December 2014.

[52] U. Banerjee, P. Bit, R. Ganguly, and S. Hardt. Aggregation dynamics of particles in a microchannel due to an applied magnetic field. Microfluid. Nanofluid., 13:565-577, 2012.

[53] R. Ganguly and I. K. Puri. Microfluidic transport in magnetic MEMS and bioMEMS. Wiley Interdiscip. Rev. Nanomed. Nanobiotechnol., 2:382-399, 2010.

[54] S. Chakraborty. Experimental characterizations of the magnetic and thermal properties of ferrofluid. Master's thesis, Jadavpur University, 2010.

[55] D. L. Youngs. Time-dependent multi-material flow with large fluid distortion. Numer. Meth. Fluid Dyn., 24:273-285, 1982.

[56] G. Y. Soh, G. H. Yeoh, and V. Timchenko. Numerical investigation on the velocity fields during droplet formation in a microfluidic T-junction. Chem. Eng. Sci., 139:99-108, 2016. 